\title{
Hacia la educación en Género: Comunicación e Igualdad
}

\author{
Carmen RodRÍGUEZ WANGÜEMERT \\ Universidad de La Laguna \\ crodrigu@ull.es \\ María del Pilar MATUd AzNAR \\ Universidad de La Laguna \\ pmatud@ull.es \\ José Manuel Pestano Rodríguez \\ Universidad de La Laguna \\ jpestano@ull.es
}

\begin{abstract}
Resumen
El presente trabajo se plantea desde el rediseño del panorama actual de los medios de comunicación con respecto al género, bajo el amparo de la Ley Orgánica 3/2007 para la Igualdad Efectiva entre hombres y mujeres. El objetivo es conocer la representación de mujeres y hombres, a través del género de quien firma los textos y las fotografías en la prensa nacional, así como el género de las fuentes citadas. Los resultados evidencian gran desigualdad. Se concluye la necesidad de eliminar la pasividad o elusión, ante la desigualdad de género, entre los futuros profesionales de la Comunicación.
\end{abstract}

Palabras clave: Educación superior; género; mujer; medios; igualdad; educomunicación.

\section{Towards Higher Education in Gender: Communication and Equality}

\begin{abstract}
This paper is presented under the re-design of the present situation of mass media with regards to gender, under the Spanish Organic Act 3/2007, for the Effective Equality between men and women. The objective is to identify the representation of women and men, through the gender of those who sign the texts and photographs in the national press, as well as the gender of the cited sources. The results show great inequality. The conclusion reached being the need to eliminate passiveness or elusion before gender equality among future professionals of Communication.
\end{abstract}

\section{Key words:}

Higher Education; gender; women; mass media; equality; educommunication.

\section{Referencia normalizada:}

Rodríguez Wangüemert, C.; Matud Aznar, M. del P. y Pestano Rodríguez, J. M. (2013) Hacia la educación en Género: Comunicación e Igualdad. Historia y Comunicación Social. Vol. 18. No Especial Noviembre. Págs. 743-755.

Sumario: 1. Introducción. 2. Género y Universidad: Grados en Comunicación. 3. La construcción de significados. 4. Diferencias de género en la profesión periodística y en las fuentes. 5. Material y Métodos. 6. Análisis y resultados. 6.1. Diferencias en función del género de quien firma. 6.2. Diferencias en las fuentes citadas. 7. Discusión y conclusiones. 8. Referencias. 


\section{Introducción}

A pesar de lograrse la igualdad jurídica formal, subsiste la desigualdad de género, tanto en el ámbito público como en el privado (INE, 2012a). La Igualdad toma forma en la sociedad de manera lenta, como un principio no del todo asimilado, para el imaginario colectivo a través de la implantación de la legislación (Ley orgánica 3/2007), pero no actúa de forma directa en la transformación real de la sociedad ni en la representación social que de la misma hacen los medios de comunicación. Es así, a pesar de que los medios son nombrados en dicha ley en el Título III, art. 36, como agentes para la divulgación sobre la Igualdad.

Esta investigación, suscrita en el marco del Proyecto de Investigación financiado por el Ministerio de Economía y Competitividad (Referencia: FEM2012-34632), desarrolla un análisis cuantitativo del contenido de la prensa española, sobre la representación de género en relación con sus profesionales periodistas y sobre las fuentes que utilizan. Además, el planteamiento del trabajo se interrelaciona con la fundamentación legislativa que implica a la Formación Superior con el principio y derecho fundamental de Igualdad (Real Decreto 1393/2007 de Enseñanzas Universitarias).

Existe evidencia empírica de que la paridad no está presente en las noticias, y que si la presencia real de las mujeres incrementa, ocupa un lugar aún ínfimo en la prensa: las noticias sobre género o protagonizadas por mujeres son el $6 \%$ de lo publicado (Bezunartea, García y Rodríguez, 2012). Las periodistas representan un $40 \%$ de las redacciones, pero sus contenidos apenas ocupan las portadas. En todos los medios son el $37 \%$, porcentaje que no ha cambiado desde 2005 . Y mientras que solo el $6 \%$ de lo publicado reta los estereotipos de género, el 46\% los refuerza (Global Media Monitoring Project, 2010). Y aunque aparenten modernidad, se ha detectado que muchas series de televisión son portadoras de discursos que reproducen la desigualdad (Belmonte y Guillamón, 2008). Se aborda la materia de género e Igualdad en la formación sobre Comunicación, como un compromiso para la mejora del reflejo de la realidad en los medios de comunicación.

Esta investigación se acerca a la estrategia universitaria y propuesta pedagógica de la comunicación para la Igualdad en el Espacio Europeo de la Educación Superior (EEES), como una manera de establecer la fundamentación crítica para el cambio social en materia de Igualdad (Gámez y Nos Aldás, 2012). Siempre, desde la perspectiva de que la formación en Comunicación influye sobre los mensajes y las estrategias para abordar temas y contenidos (Pestano, Rodríguez y Delponti, 2011).

La presente investigación presenta la novedad de acercarse al eje de responsabilidad de la Educación Superior y plantea posibilidades de cambios en las relaciones complejas entre género, producción informativa y contenidos, y se hace desde la perspectiva de los estudios sobre la representación de género en la prensa (Matud, Rodríguez y Espinosa, 2011). 


\section{Género y universidad: grados en comunicación}

En la Universidad española, según datos del INE (2012b), respecto al total de personas matriculadas el $54,2 \%$ son mujeres, y por comunidades autónomas en ninguna desciende del 52,3\%. En lo que respecta a Comunicación, de cada 100 alumnos matriculados en la Universidad española, en 2009, siete eran de Ciencias de la Comunicación.

Según indican las cifras no parecería propio plantearse el problema de la desigualdad de género. En la Universidad española, por cada 100 estudiantes varones salientes, durante el curso 2011/2012, hubo 137,1 mujeres: un 37,1\% superior. En la UE la cifra ascendió a 150,7. En Ciencias Sociales $62,4 \%$ fueron las españolas graduadas y $62,6 \%$ las de la UE.

Soriano, Cantón y Díez (2005) advertían que los datos sobre la inserción laboral de la mujer en el periodismo español, en una investigación realizada para el Collegi de Periodistas de Catalunya, señalaban la hipótesis dominante de la feminización de la profesión. Frente a ello, valoran que ha de hablarse de la pseudofeminización. Existe una desconexión, seguramente debido a la configuración del periodismo como una de las culturas dominantes. En ese mismo sentido, destaca un estudio sobre el perfil de periodistas vascos: de cada tres periodistas (el 35,2\%) una es mujer, a pesar de que durante dos décadas anteriores al análisis, el número de licenciadas fue muy superior al de licenciados (Cantalapiedra, Coca y Bezunartea, 2003).

Acercarse a las fuentes que propician la reforma de Bolonia en la Universidad en razón del género, y plantear la docencia en género en Ciencias de la Comunicación, requiere aproximarse a los planteamientos de los estudios de Comunicación en España en los últimos años. Pestano et al., (2011) señalan que dichos estudios se encuentran inmersos en transformaciones profundas que producen más interrogantes que certezas. El modelo universitario se encuentra en la dicotomía de resolver qué dirección tomar, si la propia de la sociedad del conocimiento o el enfoque de la economía del conocimiento.

En la postura crítica propuesta por Servaes (2012) sobre las tendencias en Comunicación para el Cambio Social en todo el proceso de construcción de significados sociales, surge en el presente trabajo la idea de la responsabilidad de la formación universitaria "enfocada desde la transformación socio-cultural hacia ciudadanías igualitarias, críticas y participativas, lo que reclama un proyecto educativo crítico y dialógico" (Gómez y Nos Aldás, 2012, p. 326).

\section{La construcción de significados}

Si afrontamos la propuesta de acciones, destaca un estudio sobre la producción informativa y el género, que ha iluminado alguna de las categorías del análisis de 
contenido del presente trabajo. La Prensa por dentro (Gallego et al., 2002) recoge una investigación de campo sobre el proceso de la producción de la información. Metodológicamente, aporta la novedad de la observación de profesionales el ámbito de la redacción. Y, desde esa mirada, plantea la asimetría de la representación de los géneros desde cómo los y las profesionales construyen los diferentes acontecimientos.

En ese sentido, importa especialmente la presencia en la toma de decisiones y cómo se abordan las fases de las previsiones, aspectos no perceptibles en los análisis de contenido convencionales. En el trabajo de Gallego et al., (2002) destaca que las temáticas relativas al género siempre estén en el límite de la inclusión y por tanto, también en el de la exclusión. Al respecto, se asegura que la discusión en los Consejos de Redacción no es tal, sino que se mantiene su esencia como lugar simbólico de representación jerárquica del medio.

\section{Diferencias de género en la profesión periodística y en las fuentes}

Se han encontrado diferencias en función del género en el número de profesionales que trabajan en los medios, sobre todo en los puestos de mayor poder y decisión. Cuando se analiza el organigrama directivo de la mayoría de los principales medios europeos se encuentra que hay pocas mujeres en los puestos de dirección (Areste, 2003). Hay mayor presencia de mujeres en los países nórdicos, que es donde existe mayor igualdad de género. Pero, aunque en Suecia casi la mitad de periodistas son mujeres, su presencia es mucho menor en los niveles más altos de las empresas informativas (Djerf-Pierre, 2005).

También en España son pocas las mujeres que están en los puestos de toma de decisiones de los medios (Areste, 2003), si bien hay diferencias importantes en función del medio, siendo menos las mujeres directivas en la prensa y más en televisión (Valverde, 2008).

La evidencia indica la relevancia de las características personales en cómo se cubren las noticias y una de ellas es el género (Craft y Wanta, 2004). Se ha planteado que, dado que se trata de una profesión que ha estado ampliamente dominada por hombres, el acercamiento a las noticias podría haber estado influido por ello y la perspectiva podría ser diferente en la medida en que las mujeres se incorporen a la profesión (Peiser, 2000). Pero la evidencia empírica sobre la existencia de diferencias en las noticias en función del género del redactor no es concluyente (Lavie y Lehman-Wilzig, 2005), si bien parecen existir factores mediadores de tales diferencias tales como el tamaño del medio y la composición del equipo (Craft y Wanta, 2004; Rodgers y Thorson, 2003). Además también influye el tipo y contenido de la información y la sección. En un estudio realizado en la televisión canadiense se encontró que era más común en las mujeres que en los hombres periodistas informar de noticias generales y "blandas" (Soderlund, Surlin y Romanow, 1989). Y en un 
análisis de programas informativos de tres televisiones de carácter local de Estados Unidos, se encontró que era más frecuente que las mujeres reporteras informasen de historias de interés humano y relacionadas con la salud, mientras que era más común que los hombres informasen de política (Desmond y Danilewicz, 2011). Además, tradicionalmente, la sección deportiva ha estado dominada por los hombres $\mathrm{y}$, aunque en los últimos años se ha detectado una mayor tolerancia hacia la presencia de mujeres periodistas, los hombres siguen predominando en los puestos de decisión (Hardin y Shain, 2005).

Además, las mujeres reporteras se han convertido en modelos de rol poderosos para las audiencias femeninas (Mack, 2003). Se ha planteado que la implicación de las mujeres en las noticias es un área importante de investigación por varias razones (Desmond y Danilewicz, 2011):

1. Si se encuentran diferencias sistemáticas en función del género en el tipo de noticias de las que se informa, las audiencias pueden esperar que solo determinadas áreas son apropiadas para un determinado género. Y si las jóvenes no se ven con roles importantes en los medios no se decidirán a entrar en una profesión en la que no pueden desarrollar todos sus talentos.

2. Las mujeres que se incorporan a la profesión periodística ganarán confianza en la carrera elegida en la medida en que vean a otras mujeres en posiciones visibles y en nuevos programas. Y si las mujeres profesionales son consideradas como válidas solo para determinados tópicos estereotipados, las audiencias aceptarán que esa limitación es la norma.

3. No es bueno para la profesión periodística que haya sesgos de género en la asignación de los temas. Si son asignados de forma estereotipada en función del género, en lugar de por el grado de experiencia o por cualificación, la calidad de las noticias se deteriorará.

Otra de las áreas en el estudio de las diferencias de género en la información ha sido el género de las fuentes utilizadas, aspecto que se considera muy importante, no solo en la construcción de la noticia, sino también en su orientación y en la perspectiva desde la que se realiza (Armstrong, 2006; Ross, 2007).

Aunque existe evidencia empírica de que es más probable que sean citados como fuentes los hombres que las mujeres (Armstrong, 2006; Freedman y Fico, 2005; Ross, 2007; Zeldes y Fico, 2005), esta tendencia parece disminuir cuando las redactoras son mujeres, existiendo algunos estudios que muestran que las redactoras utilizan más mujeres como fuentes que sus colegas masculinos (Freedman y Fico, 2005; Zeldes y Fico, 2005; Zoch y Turk, 1998). Pero la evidencia no es completa, existiendo estudios en los que no se ha encontrado que sea más frecuente que recurran a otras mujeres como fuentes las mujeres periodistas frente a sus colegas masculinos (Ross, 2007).

Además, abordamos cómo está representado actualmente el género en la prensa nacional española dados los cambios sociales y legislativos de los últimos años. Entre ellos destacan la Ley Orgánica 1/2004, de 28 de diciembre, de Medidas de Protección 
Integral contra la Violencia de Género, que dedica el Capítulo II a la publicidad y los medios de comunicación y donde se recoge que "fomentarán la protección y salvaguarda de la igualdad entre hombre y mujer" (p. 4217); y la Ley Orgánica 3/2007, de 22 de marzo, para la igualdad efectiva entre mujeres y hombres, cuyo Título III se titula "Igualdad y medios de comunicación" y donde se afirma que "todos los medios de comunicación respetarán la igualdad entre mujeres y hombres" (p. 12619). Los objetivos específicos son los siguientes:

1. Revisar si hay diferencias en función del género en la firma de los textos y fotografías publicadas.

2. Conocer si hay diferencias en función del género en las fuentes citadas.

3. Analizar si el género de quien firma el texto se asocia con el género de la fuente citada.

\section{Material y métodos}

Los datos se obtuvieron a partir del análisis de contenido de un diario obtenido del muestreo aleatorio de los diarios nacionales españoles de información general de mayor difusión. Para obtener una muestra representativa del contenido en los distintos meses del año y días de la semana se siguió la técnica de la "semana construida". Para ello se eligió un día de la semana y un mes del año de forma aleatoria y se seleccionó el primer periódico para el análisis. La elección del resto fue a través de la selección de un periódico cada mes eligiendo el día de la semana consecutivo al del mes anterior de forma que se completasen los siete días de la semana. De este modo se analizaron 14 periódicos, que corresponden a catorce meses diferentes y en los que se cuenta con dos números de cada día de la semana. El primero se publicó el lunes 1 de marzo de 2010 y el último al domingo 10 de abril de 2011. Se realizó un análisis del contenido de todo el periódico, a excepción de los anuncios clasificados, las viñetas humorísticas, las esquelas, la información meteorológica, la programación de televisión y las cartas al director.

\section{Análisis y resultados}

\subsection{Diferencias en función del género de quien firma}

Se encontraron un total de 2082 unidades susceptibles de que fuese publicado el nombre de su autor o autora. Un total de 1214, que suponen el 58,3\%, corresponden a textos, 843 a fotografías (el 40,5\%), y 25 son ilustraciones (el 1,2\%). 
En la tabla 1 se muestran los resultados del análisis de contingencias entre el tipo de contenido y el género de la persona firmante. Las diferencias entre los porcentajes son estadísticamente significativas, $\chi^{2}(8, N=2082)=151,74, p<.001$. Como puede observarse, un total de 794 unidades, que corresponden al 38,1\% del total, no estaban firmadas. En 218, que suponen el 10,5\%, estaban las iniciales o el pseudónimo por lo que tampoco era evidente el género de su autor o autora. Firmadas por un hombre había 819 , que suponen el 39,3\% del total de unidades publicadas; la firma de una mujer aparecía en 217, que representan el 10,4\%; y la autoría de personas de ambos géneros constaba en 34 unidades, que representan el 1,6\% del total.

Como puede observarse en la Tabla 1, los hombres aparecían identificados como autores con mucha mayor frecuencia que las mujeres, tanto de los textos (el 42,4\%) como de las fotografias (el 34,8\%) y de las ilustraciones publicadas (el 44\%), mientras que el nombre de las mujeres como autoras solo aparecía en el 15,1\% de los textos y en el $4 \%$ de las fotografías.

Tabla 1: Análisis de contingencias entre el tipo de contenido y el género de la persona firmante

\begin{tabular}{|c|c|c|c|c|c|c|c|}
\hline \multirow[b]{2}{*}{ Firmante } & \multicolumn{2}{|c|}{ Textos } & \multicolumn{2}{|c|}{ Fotografías } & \multicolumn{2}{|c|}{ Ilustraciones } & \multirow[b]{2}{*}{ Total } \\
\hline & $\mathrm{N}$ & $\%$ & $\mathrm{~N}$ & $\%$ & $\mathrm{~N}$ & $\%$ & \\
\hline Sin firma & 388 & 32,0 & 401 & 47,6 & 5 & 20,0 & 794 \\
\hline Hombre & 515 & 42,4 & 293 & 34,8 & 11 & 44,0 & 819 \\
\hline Mujer & 183 & 15,1 & 34 & 4,0 & 0 & 0 & 217 \\
\hline Hombre y mujer & 33 & 2,7 & 1 & 0,1 & 0 & 0 & 34 \\
\hline No identifica género & 95 & 7,8 & 114 & 13,5 & 9 & 36,0 & 218 \\
\hline
\end{tabular}

En 111 de los textos aparecía la foto de su autor o autora. En 97 casos, que suponen el $87,4 \%$, se trataba de un hombre y en 14 (el 12,6\%) era una mujer. También se quiso conocer si existían diferencias en la extensión de los textos publicados en función del género de quien firmaba, para lo que se hicieron Análisis de Varianza multivariados (MANOVA) y univariados (ANOVA). El MANOVA en el que se consideró como factor el género de la persona que aparecía como firmante y como variables dependientes el número de columnas y el número de líneas ocupado por los respectivos textos evidenció que no existían diferencias estadísticamente significativas, $F(2,695)$ $=2,33, p<.05$. Tampoco se encontraron diferencias estadísticamente significativas en los ANOVAs realizados para comparar si había diferencias en el número de filas y en el de columnas ocupadas por dichos textos (véase Tabla 2). 
Tabla 2. Medias, desviaciones típicas y análisis de varianza en la extensión de los textos según el género de quien los firma

\begin{tabular}{|l|l|l|l|l|l|}
\hline & \multicolumn{4}{|l|}{ Hombre $(\mathrm{N}=515)$} & \multicolumn{2}{l|}{ Mujer $(\mathrm{N}=183)$} & $F$ \\
\hline Extensión & $M$ & $D T$ & $M$ & $D T$ & $(1,696)$ \\
\hline $\mathrm{N}^{\circ}$ de columnas & 3,82 & 1,96 & 4,15 & 2,00 & 3,76 \\
\hline $\mathrm{N}^{\mathrm{o}}$ de líneas & 64,77 & 35,06 & 64,52 & 345,84 & 0,01 \\
\hline
\end{tabular}

También se realizó análisis de contingencias para conocer si existían diferencias en función del género de la persona autora del texto y la sección del periódico en que aparecía publicado dicho texto, datos que se muestran en la Tabla 3. Las diferencias entre los porcentajes fueron estadísticamente significativas, $\chi^{2}(8, N=698)=57,30, p$ $<.001$. Como puede observarse, en todas las secciones es más común que aparezcan los hombres como firmantes de los textos que las mujeres, si bien la magnitud de las diferencias depende de la sección. Donde mayor desigualdad se detectó fue en Opinión donde el 90,3\% de los textos de esta sección estaban firmados por hombres y solo el $9,7 \%$ por mujeres; le sigue Deportes, donde el $89,9 \%$ de los textos los firman hombres y el 10,1\% mujeres; Cultura con el $82,2 \%$ de textos firmados por hombres y el $17,8 \%$ por mujeres; Sociedad donde el $81,1 \%$ de los textos de esta sección están firmados por hombres y el $18,9 \%$ por mujeres; y Contraportada con un $80 \%$ de textos firmados por hombres y el $20 \%$ por mujeres.

Tabla 3 Género de la persona firmante y sección del periódico donde aparece el texto

\begin{tabular}{|l|l|l|l|l|l|}
\hline & \multicolumn{2}{|c|}{ Hombre } & \multicolumn{2}{l|}{ Mujer } & \\
\hline Sección & $\mathrm{N}$ & $\%$ & $\mathrm{~N}$ & $\%$ & Total \\
\hline Opinión & 93 & 18,1 & 10 & 5,5 & 103 \\
\hline Nacional & 84 & 16,3 & 50 & 27,3 & 134 \\
\hline Internacional & 56 & 10,9 & 33 & 18,0 & 89 \\
\hline Sociedad & 30 & 5,8 & 7 & 3,8 & 37 \\
\hline Economía & 48 & 9,3 & 30 & 16,4 & 78 \\
\hline Deportes & 62 & 12,0 & 7 & 3,8 & 69 \\
\hline Cultura & 97 & 18,8 & 21 & 11,5 & 118 \\
\hline Portada & 25 & 4,9 & 20 & 10,9 & 45 \\
\hline Contraportada & 20 & 3,9 & 5 & 2,7 & 25 \\
\hline
\end{tabular}

\subsection{Diferencias en las fuentes citadas}

En la mayoría de los textos (el 93,2\%) no se citó a ninguna fuente. Cuando sí se citaron fuentes lo más común es que se tratase de un hombre experto, lo que se dio en 
el $68,7 \%$ de los casos en que se citó alguna fuente; mujeres expertas se citaron en el $10,8 \%$; hombres testigos en el 9,6\%; mujeres testigos en el 6,1\% y personas de ambos géneros en calidad de testigo en el $4,8 \%$. Las diferencias entre los porcentajes fueron estadísticamente significativas, $\chi^{2}(4, N=83)=123,93, p<.001$.

Para conocer si había asociación sistemática entre el género de fuentes citadas y el género de la persona firmante del texto se hizo análisis de contingencia, datos que se muestran en la tabla 4 . Las diferencias de porcentajes no fueron estadísticamente significativas, $\chi^{2}(4, N=68)=7,58, p>.05$, aunque debido a que 6 de las casillas, que suponen el $60 \%$, tienen frecuencias esperadas menores que 5 , el estadístico de contraste es poco fiable.

Tabla 4 Género de la persona firmante y género de la fuente citada

\begin{tabular}{|l|l|l|l|l|l|}
\hline & \multicolumn{4}{|l|}{ Hombre firma } & \multicolumn{2}{l|}{ Mujer firma } & \\
\hline Fuente & $\mathrm{N}$ & $\%$ & $\mathrm{~N}$ & $\%$ & Total \\
\hline Hombre experto & 93 & 18,1 & 10 & 5,5 & 103 \\
\hline Mujer experta & 84 & 16,3 & 50 & 27,3 & 134 \\
\hline Hombre testigo & 56 & 10,9 & 33 & 18,0 & 89 \\
\hline Mujer testigo & 30 & 5,8 & 7 & 3,8 & 37 \\
\hline Ambos géneros testigo & 48 & 9,3 & 30 & 16,4 & 78 \\
\hline
\end{tabular}

\section{Discusión y conclusiones}

Los resultados del presente trabajo evidencian que sigue persistiendo la desigualdad en la prensa española en la representación de mujeres y hombres. De los resultados relativos a las diferencias en la firma destaca que, mientras que era hombre quien firmaba en el 39\% de todas las unidades del periódico, la firma de una mujer solo constaba en el 10,4\% del total. Estas diferencias se extendían a las secciones del periódico. Donde más desigualdad de género se encontró fue en las más prestigiadas, tales como Opinión, o en las consideradas tradicionalmente masculinas, como Deportes. Aunque la existencia de diferencias en función del género en la profesión periodística es un tópico sujeto a debate y en el que parece haberse dado cambios en algunos países en los últimos tiempos (Hardin y Shain, 2005; Lavie y Lehman-Wilzig, 2005), también en otros estudios se ha detectado la existencia de diferencias en función del género en el tipo de noticias cubiertas (Desmond y Danilewicz, 2011; Hardin y Shain, 2005; Soderlund et al., 1989).

La desigualdad de género se detecta también en las fuentes de información, donde es mucho más común que aparezcan hombres que mujeres, sobre todo cuando se trata de personas expertas. Y esto último no parece mejorar cuando quien firma la información es una mujer. También en estudios realizados en otros países se ha encontrado 
que es más común que los hombres sean citados como fuentes respecto a las mujeres (Armstrong, 2006; Freedman y Fico, 2005; Ross, 2007; Schwartz, 2011; Zeldes y Fico, 2005).

Cabe plantearse la necesidad de mejorar la enseñanza en género en Comunicación, porque la realidad numérica refleja que no está representada la mitad de la población presente en la sociedad (al menos el 50\% es mujer), y por tanto eso significa que no se hallan reflejadas en los discursos informativos esas ciudadanas.

La atención al género en la Enseñanza Superior está pendiente de fraguarse en competencias académicas efectivas. Todo apunta a la necesidad de propuestas de actuación críticas que van desde el propio lenguaje de los discursos hasta la aplicación de actividades para la consecución de resultados en la pragmática de la enseñanza universitaria, porque todo proceso de educación es también un proceso de comunicación (López y Encabo, 2008).

Todo revela la necesidad de cambios aunque los resultados de un estudio centrado en las demandas de los propios medios de comunicación a las universidades, no satisfagan los planteamientos de la presente investigación. Al respecto señalan Vázquez y Fernández (2012) que desde el sector profesional de la Comunicación no existe ninguna demanda a las universidades de formación relacionada con conocimiento sobre género o Igualdad, cuando se les pregunta sobre los conocimientos que creen que precisan los Grados en Comunicación. Las demandas expresadas priman la necesidad de manejo de tecnologías e idiomas, nada desdeñables ni reñidas con el presente trabajo, pero que sitúan en claro peligro de exclusión las actuaciones específicas sobre género e Igualdad en los planes de estudio de Comunicación.

\section{Referencias bibliográficas}

ARESTE (2003). Arrinconando estereotipos en los Medios de comunicación y la Publicidad. Madrid: Dirección General de la Mujer.

ARMSTRONG, C. L. (2004). "The influence of reporter gender on source selection in newspaper stories". En Journalism \& Mass Communication Quarterly, n 81, p. 139-154.

BELMONTE, J.; GUILLAMÓN, S. (2008). “Co-educar la mirada contra los estereotipos de género en TV”. Comunicar, no 31, p. 115-120.

BEZUNARTEA, O; GARCÍA GORDILLO; M. M.; RODRÍGUEZ REY, A. (2012). "La mujer como cargo y como fuente en la prensa escrita. La paridad no llega a las noticias". En Ámbitos, no 21, p. 233-256.

BREA, J.L. (2005). Estudios Visuales. Madrid: Akal

CANTALAPIEDRA, M. J.; COCA, C.; BEZURNATEA, O. (2000). "La situación laboral y profesional de los periodistas". En ZER Revista de Estudios de Comunicación, $\mathrm{n}^{\mathrm{o}} 5$, p. 335-355. 
CRAFT, S.; WANTA, W. (2004). "Women in the newsroom: influences of female editors and reporters on the news agenda". En Journalism \& Mass Communication Quarterly, no 81, p. 124-138.

DESMOND, R.; DANILEWICZ, A. (2011). "Women are on, but not in, the news: Gender roles in local television news". En Sex Roles, no 62, p. 822-829.

DJERF-PIERRE, M. (2005). "Lonely at the top". En Journalism, no 6, p. 265-290.

FREEDMAN, E.; FICO, F. (2005). "Male and female sources in newspaper coverage on male and female candidates in open races for Governor in 2002". En Mass Communication \& Society, $n^{\circ} 8$, p. 257-272.

GALLEGO, J.; ALTÉS, E.; MELÚS, M. E.; SORIANO, J.; CANTÓN, M. J. (2002). La prensa por dentro. Producción informativa y transmisión de estereotipos de género. Barcelona: Los libros de la frontera.

GÁMEZ M. J.; NOS ALDÁS, E. (2012). "Comunicación para la igualdad en el nuevo EEES: fundamentación crítica para el cambio social". Estudios sobre el Mensaje Periodístico, $\mathrm{n}^{\mathrm{o}}$ 1, p. 325-335.

GLOBAL MEDIA MONITORING PROJECT (2010). Who makes the news? World Association for Christian Communication (WACC).

http://www.whomakesthenews.org/images/reports_2010/global/gmmp_global_ report_es.pdf [28.09.2013].

HARDIN, M.; SHAIN, S. (2005). "Strength in numbers? The experiences and attitudes of women in sports media careers". En Journalism \& Mass Communication Quarterly, no 82, p. 804-819.

INE (2012a). La Enseñanza Universitaria en España, curso 2010-2011. Madrid: INE. INE (2012b). Mujeres y hombres en España. Madrid: INE.

LAVIE, A.; LEHMAN-WILZIG, S. (2005). "The method is the message. Explaining inconsistent findings in gender and news production research". En Journalism, $\mathrm{n}^{\mathrm{0}}$ 6, p. 66-89.

Ley Orgánica 1/2004, de 28 de diciembre, de Medidas de Protección Integral contra la Violencia de Género. Madrid: BOE.

Ley Orgánica 3/2007, de 22 de marzo, para la igualdad efectiva entre mujeres y hombres. Madrid: BOE.

LÓPEZ VALERO, A.; ENCABO, E. (2008). Lenguaje, Cultura y Discriminación. La equidad comunicativa entre géneros. Granada: Mágina.

MACK, C. (2003). "Ann Curry: Leaving the dream". En Flash, no 18, p. 1-3.

MATUD, M. P.; RODRÍGUEZ, C.; ESPINOSA, I. (2011). "Gender in Spanish Daily Newspapers". En Sex Roles, n 64, p. 253-264.

MINISTERIO DE EDUCACIÓN, CULTURA Y DEPORTE (2013). Datos y Cifras del Sistema Universitario Español, curso 2012-2013. Madrid: MECD.

PEISER, W. (2000). "Setting the journalist agenda: Influences from journalist' individual characteristics and from media factors". En Journalism \& Mass Communication Quarterly, $\mathrm{n}^{\mathrm{o}}$ 77, p. 243-257.

PESTANO, J. M.; RODRİGUEZ WANGÜEMERT, C.; DELPONTI, P. (2011). "Transformaciones en los modelos de formación de periodistas en España. El reto europeo". Estudios sobre el Mensaje Periodístico, nº 17, p. 401-415. 
Real Decreto 1393/2007, de 29 de octubre, por el que se establece la ordenación de las enseñanzas universitarias oficiales. Madrid: BOE.

RODGERS, S.; THORSON, E. (2003). "A socialization perspective on male and female reporting". En Journal of Communication, $\mathrm{n}^{\circ}$ 53, p. 658-675

ROSS, K. (2007). "The journalist, the housewife, the citizen and the press. Women and men as sources in local news narratives". En Journalism, no 8, p. 449-473.

SCHWARTZ, J. (2011). "Whose voices are heard? Gender, sexual orientation and newspapers sources". En Sex Roles, no 64, p. 265-275.

SERVAES, J. (2012). "Comunicación para el desarrollo sostenible y el cambio social. Una visión general”, En Cuadernos de Información y Comunicación, vol. 17, p.17-40.

SODERLUND, S.; SURLIN, S.; ROMANOW, W. (1989). "Gender in Canadian local television news: Anchors and reporters". En Journal of Broadcasting \& Electronic Media, $\mathrm{n}^{\circ}$ 33, p. 187-196.

SORIANO, J.; CANTÓN, M.J.; DÍEZ, M. (2005). "La pseudofeminización de la profesión periodística en España". En ZER Revista de Estudios de Comunicación, $\mathrm{n}^{\circ}$ 19 , p. 35-52.

VALVERDE, C. (2008). "Tratamiento de la mujer en los medios". En Cuadernos de Comunicación, $\mathrm{n}^{\mathrm{o}} 2$, p. 38-49.

VÁZQUEZ GESTAL, M; FERNÁNDEZ SOUTO, M.B. (2012). Las necesidades formativas de los nuevos comunicadores según los profesionales y su reflejo en los Grados de Comunicación. Madrid: Servicio de Publicaciones de la Universidad Complutense.

ZELDES, G. A.; FICO, F. (2005). "Race and gender: An analysis of sources and reporters in the network's coverage of the 2000 presidential campaign". En Mass Communication \& Society, $\mathrm{n}^{\circ} 8$, p. 373-385.

ZOCH, L. M.; TURK, J. V. (1998). "Women making news: Gender as a variable in source selection and use". En Journalism \& Mass Communication Quarterly, n ${ }^{\circ}$ 75 , p. 762-775.

\section{Los autores}

Rodriguez Wangüemert, Carmen. Profesora Titular de Periodismo de la Universidad de La Laguna. Las principales líneas de investigación están relacionadas con la comunicación para el desarrollo, cultura desde la diversidad. Participa en proyectos multidisciplinares de I+D sobre comunicación y género, la ciudad, migración y cultura, también en colaboración con grupos de universidades africanas. Dirige Calima Red de Investigadores en Comunicación y Cultura Canarias-África.

Matud Aznar, María Pilar. Catedrática de Personalidad, Evaluación y Tratamientos Psicológicos de la Universidad de La Laguna. Doctora en Filosofía y Letras (Sección de Psicología). Doctora en Ciencias de la Información. Directora de la I y II Edición del Máster Universitario en Prevención e Intervención en Violencia de Género y de la 
I y II Edición del Experto/a en Intervención en Violencia de Género y del Especialista Universitario en Peritaje psicológico en Violencia de Género y en Familia. Autora o coautora de 7 libros, 27 capítulos de libros, 62 artículos publicados en revistas científicas. Investigadora en 18 Proyectos de Investigación y en 5 contratos financiados por entidades públicas.

Pestano Rodríguez, José Manuel. Profesor asociado de universidad. Sus líneas de trabajo se refieren al papel que desempeñan los medios de comunicación en la sociedad y la educación, así como al estudio de las tecnologías emergentes. Miembro de Calima Red de Investigadores en Comunicación y Cultura Canarias-África ha formado parte de varios proyectos de investigación referidos a estos temas, de los que se han derivado artículos y capítulos de libros. 